\title{
Conclusion not Yet Available
}

National Cancer Institute

\section{Source}

National Cancer Institute. Conclusion not Yet Available. NCI Thesaurus. Code C91868.

A conclusion has yet to be established as the investigation is incomplete. 